\title{
Incidencia de tuberculosis en pacientes con psoriasis que reciben terapias anti- TNF- alfa en Latinoamérica: revisión sistemática y metaanálisis

\author{
Tuberculosis incidence in patients with psoriasis who receive anti- TNF- alpha \\ therapies in Latin America: systematic review and meta-analysis
}

\author{
Roniel Gonzalo Contreras Maza ${ }^{1, a}$ \\ ${ }^{1}$ Servicio de Dermatología, Clínica Ricardo Palma. Lima, Perú. \\ a Médico dermatólogo, ORCID: https://orcid.org/0000-0002-5431-1598
}

An Fac med. 2019; 80(1):73-8. / DOI: https://doi.org/10.15381/anales.v80i1.15623

\begin{abstract}
Correspondencia:
Roniel Gonzalo Contreras Maza

ronielgcm@gmail.com
\end{abstract}

Recibido: 26 de diciembre 2018 Aprobado: 15 de febrero 2019

Publicación en línea: 27 de marzo 2019

Conflictos de interés: El autor declara no tener conflictos de interés

Fuente de financiamiento: Autofinanciado

Citar como: R. Incidencia de tuberculosis en pacientes con psoriasis que reciben terapias antiTNF- alfa en Latinoamérica: revisión sistemática y metaanálisis. An Fac med. 2019;80(1):73-8. DOl: https://doi. org/10.15381/anales.v80i1.15623

\section{Resumen}

La incidencia global de tuberculosis en pacientes con psoriasis recibiendo anti-factor de necrosis tumoral (TNF)- $\alpha$ en Latinoamérica es desconocida, a pesar del uso cada vez mayor de dichas terapias, y de las elevadas tasas de incidencia de tuberculosis en la región, por lo que se efectuó una revisión sistemática y metaanálisis sobre el tema. Para dicha revisión, se incluyeron estudios que reporten la incidencia de tuberculosis activa en pacientes con psoriasis recibiendo anti- TNF- $\alpha$ en Latinoamérica, usando cuatro bases de datos electrónicas: Pubmed, Scielo, LILACS y Medigraphic, para luego efectuar un metaanálisis sobre dichas incidencias y obtener proporciones conjuntas. Finalmente, se seleccionaron 9 estudios los cuales fueron realizados en Argentina, Brasil, Chile, Colombia y México, con un seguimiento total de 510,9 pacientes- año, y obteniéndose un total de 3 casos de tuberculosis activa, lo cual constituye una incidencia estimada de 636 casos de tuberculosis por cada 100 mil pacientes/año (intervalo de confianza al 95\% [IC 95\%]: 145-1764 por 100 mil pacientes/año), tras usar el modelo de efectos fijos, siendo dicha incidencia elevada en relación a las tasas de incidencia poblacionales en la región, y comparable a otras poblaciones que recibieron terapias anti- TNF- $\alpha$ por psoriasis en otras latitudes.

Palabras clave: Tuberculosis; Psoriasis; Etanercept; Infliximab; Adalimumab

\section{Abstract}

The global incidence of tuberculosis in patients with psoriasis who receive anti- tumoral necrosis factor (TNF)alpha in Latin America remains unknown, despite of the increasing use of those therapies and the local high incidences of tuberculosis, so a systematic review and meta-analysis on the subject was carried out. For that review, there were included studies reporting the tuberculosis incidence in patients with psoriasis receiving anti- TNF- alpha in Latin America, using four electronical databases: Pubmed, Scielo, LILACS and Medigraphic, and then to perform a meta-analysis about those incidences in order to obtain pooled proportions. Finally, 9 studies were selected, which were performed in Argentina, Brasil, Chile, Colombia and México, with a total follow-up of 510,9 patient-years, and with three cases of tuberculosis, giving a pooled incidence of 636 cases of tuberculosis per 100,000 patient-years (95\% confidence interval [CI 95\%]: 145-1764 per 100,000 patient-years), after using the fixed effects model, which was high according to the stimates of the regional population- based tuberculosis incidence, and similar to other studies performed in patients with psoriasis who receive anti- TNF- alpha in different locations.

Keywords: Tuberculosis; Psoriasis; Etanercept; Infliximab; Adalimumab 


\section{INTRODUCCIÓN}

La tuberculosis es una enfermedad infecciosa causada por Mycobacterium tuberculosis, la cual es de gran morbimortalidad, y cuya incidencia para el año 2017, según la Organización Mundial de la Salud (OMS) a nivel mundial, y en las Américas, fue de 133 y 28 casos por 100 mil habitantes, respectivamente ${ }^{1}$. En Latinoamérica y el Caribe, se estima que dicha incidencia para el año 2016, fue de 41 casos por 100 mil habitantes ${ }^{2}$. Además, la tuberculosis se encuentra dentro de las 10 primeras causas de mortalidad a nivel mundial ${ }^{1}$. Por otro lado, la psoriasis, es una enfermedad inflamatoria crónica cuya característica más importante es la hiperproliferación de los queratinocitos, y que se manifiesta principalmente por lesiones cutáneas, incluyendo placas, pústulas y/o pápulas, además de otras formas clínicas menos frecuentes ${ }^{3}$. La prevalencia de psoriasis a nivel mundial es de 0,11 a 8\% en población general ${ }^{4}$, en tanto que en Latinoamérica es de $2,14 \%{ }^{5}$. Dentro de los tratamientos para la psoriasis es cada vez más frecuente el uso de terapia biológica, y dentro de ella, las terapias anti- factor de necrosis tumoral (TNF)- $\alpha$, son una de las más usadas, especialmente en nuestro país ${ }^{6}$. El riesgo de tuberculosis activa en pacientes recibiendo terapia anti-TNF- $\alpha$, ya sea por múltiples indicaciones o por psoriasis, es aún controversial ${ }^{7-10}$, aunque algunos estudios encontrarían una tendencia al aumento de la misma, especialmente si no se controlan factores de riesgo como la tuberculosis latente ${ }^{8}$. Además, parece haber un mayor riesgo de tuberculosis activa en pacientes recibiendo infliximab o adalimumab si se les compara con etanercept ${ }^{11}$. La incidencia global de tuberculosis activa en pacientes con psoriasis recibiendo antiTNF- $\alpha$ en Latinoamérica es desconocida, especialmente si se incluyen cohortes de pacientes además de los ensayos clínicos, dado que estos expresarían mejor esta incidencia. Además, muchos países de la región tienen incidencias medias a altas de tuberculosis, por lo que el objetivo principal de este estudio fue determinar la incidencia de tuberculosis en pacientes que reciben terapia anti-TNF- $\alpha$ por psoriasis en Latinoamérica.

\section{MÉTODOLOGÍA DE BÚSQUEDA Y SELECCIÓN DE RESULTADOS}

Realizamos esta revisión sistemática y metaanálisis de acuerdo a las guías MOOSE para estudios observacionales ${ }^{12}$. Se realizó una revisión sistemática de la incidencia de tuberculosis activa en pacientes con psoriasis recibiendo terapia anti-TNF- $\alpha$ en Latinoamérica usando cuatro bases de datos electrónicas: Pubmed, Scielo, LILACS y Medigraphic. Los términos de búsqueda fueron "psoriasis y biológicos", "psoriasis y anti TNF", "psoriasis e infliximab", "psoriasis y adalimumab", "psoriasis y etanercept", "psoriasis y golimumab", "psoriasis y certolizumab", añadiéndoles al final el término "tuberculosis" o "seguridad", y en las bases de datos que lo permitían se colocó el filtro para investigaciones en humanos. Esto dio un total de 2623 artículos (Pubmed 2261, Scielo 82, LILACS 234, y Medigraphic 46). Los idiomas en los que se recogieron los artículos fueron en inglés, español y portugués. Se analizaron los estudios hasta el 08 de octubre de 2018.

Se incluyeron estudios originales en seres humanos, realizados en Latinoamérica, en pacientes mayores de 18 años, con diagnóstico de psoriasis, tratados al menos 12 semanas con terapia anti- TNF$\alpha$ (no se consideraron los tratados con biosimilares), que tengan datos sobre tuberculosis o seguridad, y que incluyan el tiempo de seguimiento realizado. Se eligieron estudios con población superior a 5 personas. Las publicaciones duplicadas fueron descartadas. Asimismo, estudios de pacientes previamente tratados con biológicos fueron excluidos. Los estudios que no reportaron datos suficientes para calcular la incidencia o en los que no se pudo acceder al texto completo también fueron descartados. Los artículos fueron evaluados según el título y el resumen. Aquellos con título y resúmenes relevantes o artículos sin resumen, pero con título relevante fueron elegidos para ser evaluados a texto completo. En aquellos artículos de interés sin los datos suficientes o claros para el cálculo de la incidencia, se contactó a los autores por correo electrónico. Una vez seleccionados los artículos, se determinó las características de la población de los mismos, el tipo y duración del estudio, incidencia de tu- berculosis, cribado de tuberculosis latente, tipo de tuberculosis activa (pulmonar, extrapulmonar, o ambos). Para evaluar la calidad de los estudios se usó la escala de Newcastle- Otawa para estudios observacionales, especialmente la indicada para estudios de cohortes, o modificada, cuando los estudios eran sólo longitudinales $^{13}$, así como la herramienta de la colaboración Cochrane para evaluar riesgo de sesgos en ensayos clínicos ${ }^{14}$.

El análisis estadístico se realizó utilizando el paquete estadístico MedCalc, versión 18.11 (2018), y Microsoft Excel (2013). Para el cálculo del metaanálisis de proporciones, dentro del paquete estadístico, se usó el método de transformación de Freeman-Tukey para transformar proporciones ${ }^{15}$, luego se calculó la heterogeneidad usando el estadístico $\mathrm{I}^{2}$, así como la proporción combinada bajo el modelo de efectos fijos y aleatorios de DerSimonian y Lair ${ }^{16}$. El metaanálisis de proporciones obtuvo resultados combinados con intervalos de confianza al 95\%.

Tras usar los términos de búsqueda antes explicados se obtuvo un total de 2623 artículos. Tras aplicar el diagrama de flujo PRISMA $^{17}$ se seleccionaron 9 artículos para el presente metaanálisis (Figura 1).

\section{DESARROLLO DEL TEMA}

Los 9 estudios seleccionados fueron realizados en Argentina, Brazil, Chile, Colombia, y México, de los cuales en 4 se usaron infliximab, adalimumab y etanercept, en 4 sólo infliximab, y uno sólo usó etanercept, tanto para poblaciones con psoriasis en placas y/o artropatía psoriática principalmente. El seguimiento promedio fue de 56,8 pacientes/año (IC95\%: $21,6-91,9)^{18-21}$. El resto de datos demográficos y características de los estudios se muestran en las tablas 1 y 2 .

La incidencia promedio de tuberculosis activa fue de 636 por 100 mil pacientes/año (IC95\%: 145 a 1764 por 100 mil pacientes/ año) tal como se muestra en la figura 2. De los tres casos de tuberculosis encontrados, dos fueron de forma pulmonar y uno extrapulmonar; recibiendo los dos primeros etanercept, y, el último, adalimumab ${ }^{19}$. La mayoría de los estudios fue de tipo longitudinal $(77,8 \%)$, el 


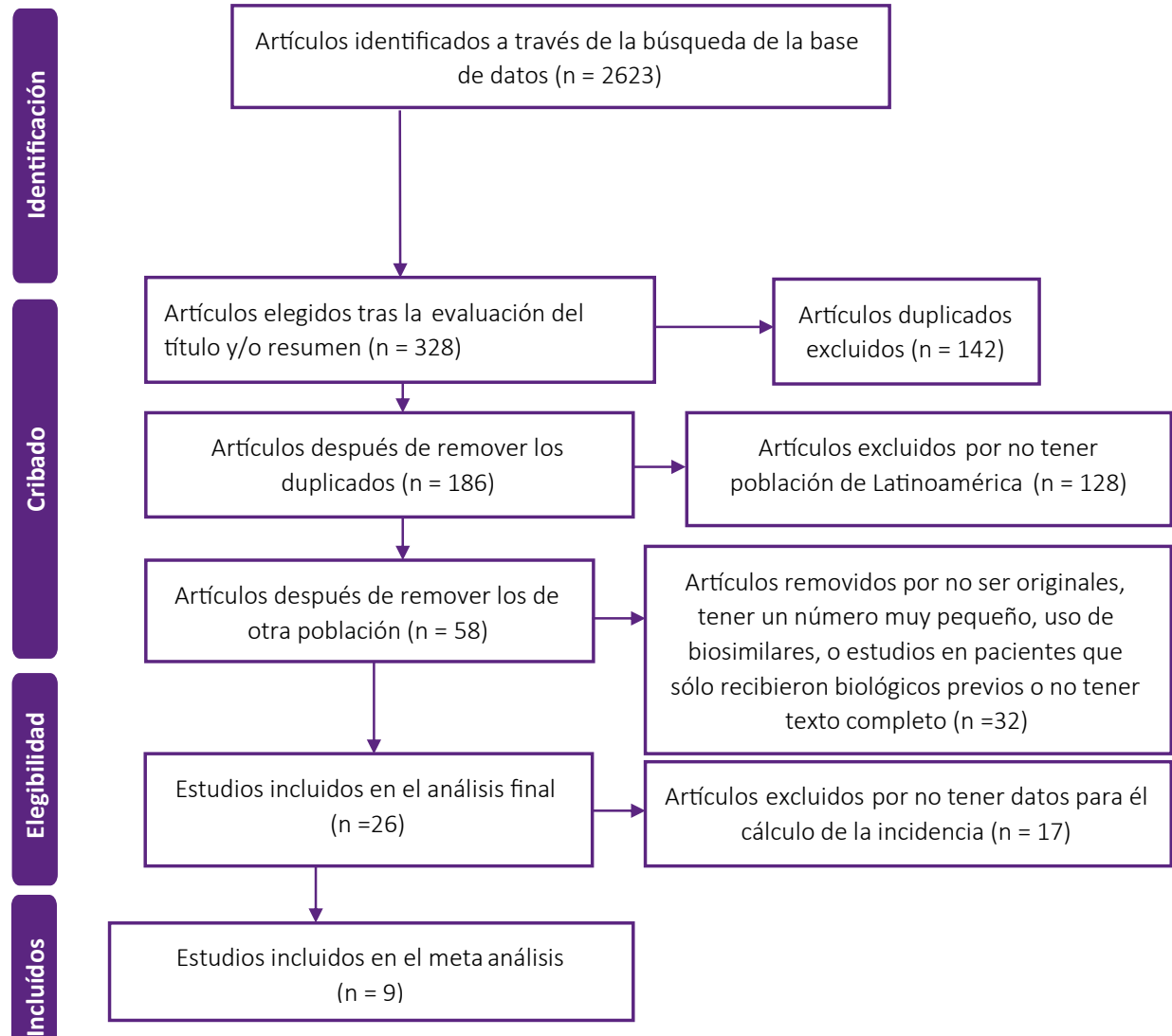

Figura 1. Diagrama de flujo PRISMA ${ }^{17}$ para la selección de estudios de incidencia de tuberculosis en pacientes con psoriasis recibiendo anti-TNF- $\alpha$. Adaptado de: Moher D, Liberati A, Tetzlaff J, Altman DG, The PRISMA Group (2009). Preferred Reporting Items for Systematic Reviews and Meta-Analyses: The PRISMA Statement.

PLoS Med. 6(7): e1000097. DOI: https://doi.org/10.1371/journal.pmed.1000097

$11,1 \%$ fue de cohortes, y el $11,1 \%$ de tipo ensayo clínico, con edades promedio que oscilan entre los 38 y 56,2 años. Cabe resaltar que sólo 7 de los 9 estudios realizaron cribado para tuberculosis latente, y en los 2 restantes no se reportaron datos respecto a dicha medida. En 4 de dichos 7 estudios se incluyeron a los pacientes con tuberculosis latente, en 2 no, y en uno no se informa al respecto. En 3 de los cuatro estudios que sí se incluyeron explícitamente a pacientes con tuberculosis latente, se reportaron frecuencias de tuberculosis latente de 18,8 a 100\%, mientras que en el estudio restante no se reportó.

En la presente revisión encontramos una incidencia combinada de 636 casos de tuberculosis activa por 100 mil pacientes/año en Latinoamérica, la cual es muy superior a la incidencia esperada para dicha población en 2016, la cual fue de 41 casos por cada 100 mil pacientes/año². Cabe resaltar que las incidencias antes señaladas son para población general, a

diferencia de las poblaciones de los estudios de este metaanálisis, que son poblaciones adultas, y, en varios casos, la po-

Andrade, 2014

Caltaño, 2016

Duarte, 2011

Gomes, 2015

Labarca, 2003

Machado, 2013

Scali, 2005

Valdés, 2006

Valenzuela, 2017

Total (fixed effects) Total (random effects)

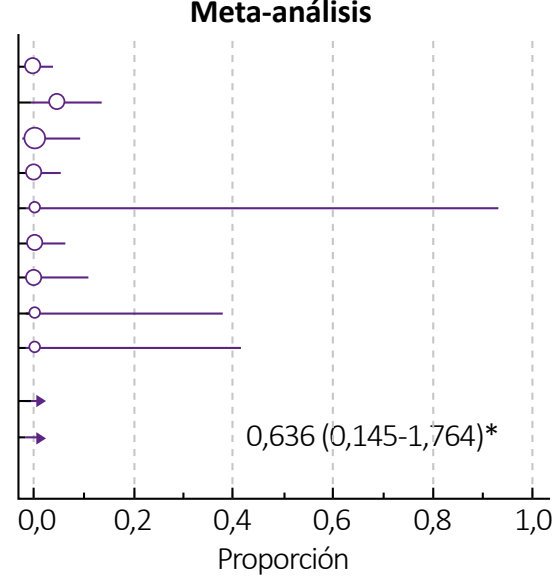

Figura 2. Estudios seleccionados y metaanálisis de la incidencia de tuberculosis en pacientes con psoriasis recibiendo anti-TNF- $\alpha$ en Latinoamérica.

*Proporción (intervalo de confianza al 95\%) 
Tabla 1. Características demográficas e incidencia de tuberculosis activa de los estudios seleccionados para el metaanálisis de incidencia de tuberculosis en pacientes con psoriasis recibiendo anti-TNF- $\alpha$ en Latinoamérica

\begin{tabular}{|c|c|c|c|c|c|}
\hline $\begin{array}{ll}\text { Autor, } & \text { año } \\
\text { [referencia] }\end{array}$ & $\begin{array}{l}\text { Casos } \\
\text { de TBC }\end{array}$ & $\begin{array}{l}\text { Seguimiento } \\
(p / a)\end{array}$ & $n(\% V)$ & Edad () & $\begin{array}{l}\text { Países en los que se } \\
\text { realizó el estudio }\end{array}$ \\
\hline Andrade, $2014^{18}$ & 0 & 111 & $74(55,4)$ & 47,7 & Brasil \\
\hline Cataño, $2016^{19}$ & 3 & 63,6 & $86\left(56,4^{*}\right)$ & $46,9 *$ & Colombia \\
\hline Duarte, $2011^{20}$ & 0 & 164,3 & $53(75,5)$ & 49,2 & Brasil \\
\hline Gomes, $2015^{21}$ & 0 & 64 & $16(43,8)$ & 56,2 & Brasil \\
\hline Labarca, $2003^{22}$ & 0 & 1,4 & $7(85,7)$ & 44,7 & Chile \\
\hline Machado, $2013^{23}$ & 0 & 60 & $12\left(35,8^{*}\right)$ & $47,5^{*}$ & Brasil \\
\hline Scali, $2005^{24}$ & 0 & 32 & $16(56,3)$ & 38,0 & Argentina \\
\hline Valdés, $2006^{25}$ & 0 & 7,7 & $8(62,5)$ & 46,0 & Chile \\
\hline Valenzuela, $2017^{26}$ & 0 & 6,9 & $30(76,7)$ & 43,8 & $\begin{array}{l}\text { Argentina, Chile y } \\
\text { México }\end{array}$ \\
\hline
\end{tabular}

* Datos que corresponden al total de participantes en el estudio, entre los que se incluyen los pacientes recibiendo anti-TNF- $\alpha$. TB: tuberculosis activa; p/a: pacientes/año; $n(\% \mathrm{~V})$ : tamaño de muestra (porcentaje de varones); $\overline{\mathrm{x}}$ : promedio

blación de estudio fue exclusivamente de pacientes con artropatía psoriática (4 de los 9 estudios), y/ o recibió medicación sistémica inmunosupresora concomitante con el anti-TNF- $\alpha$ (5 de los 7 estudios en los que se reporta este dato, pues en 2 no se especifica y en 2 no se recibieron otras terapias inmunosupresoras), lo que es preciso tomar en cuenta al momento de comparar las incidencias, aunque aun así la diferencia de las incidencias es alta.

Si se compara la incidencia del presente estudio con otras poblaciones de pacientes recibiendo anti-TNF- $\alpha$ por psoriasis, vemos, por ejemplo, que es ligeramente superior a la incidencia encontrada en un estudio efectuado en registros nacionales de España, Italia e Israel $\left.\right|^{9}$, la cual fue de 510,2 por 100 mil pacientes/ año, teniendo dichos países incidencias bajas de tuberculosis $(10,6,9$ y 3,2 por 100 mil pacientes/año para 2017) ${ }^{27}$. Además, la población de dicho estudio no recibió terapia sistémica concomitante a diferencia de nuestro estudio; $y$, en el caso de Italia, un estudio sobre una base de un registro nacional obtuvo una frecuencia de tuberculosis latente de 5,8\% para los pacientes recibiendo anti-TNF- $\alpha$ por psoriasis (del total de pacientes con historia negativa de tuberculosis) ${ }^{28}$, y otro estudio obtuvo una frecuencia de $20,5 \%$ de tuberculosis latente para España, también sobre la base de un registro nacional (para los biológicos empleados, entre los que se incluyen los anti-TNF- $\alpha)^{29}$, las cuales son bajas en comparación a nuestro estudio (48,1\% en los 3 estudios que incluyeron a pacientes con tuberculosis latente y que reportaron dicho dato). Por otro lado, un estudio realizado en Turquía, determinó una incidencia superior de tuberculosis activa, la cual fue de 890 por 100 mil pacientes/año, teniendo dicho país una incidencia de tuberculosis activa de 28 por 100 mil pacientes/año ${ }^{10}$. Dicho estudio también aceptó pacientes con medicaciones concomitantes como corticoides sistémicos, metotrexato y ciclosporina, y tuvo una tasa alta de tuberculosis latente, la cual fue de $70,6 \%{ }^{10}$, superior a la frecuencia de tuberculosis latente de la presente revisión, reafirmando la importancia de la tuberculosis latente como factor de riesgo a tener en cuenta para la reactivación tuberculosa, especialmente si esta infección es reciente ${ }^{30}$, o si se añaden factores de riesgo como terapia anti-TNF- $\alpha$, medicación inmunosupresora concomitante ${ }^{7}, y$, especialmente si no hay un manejo adecuado de

Tabla 2. Tipo, calidad y características principales de los estudios seleccionados para el metaanálisis de incidencia de tuberculosis en pacientes con psoriasis recibiendo anti-TNF- $\alpha$ en Latinoamérica

\begin{tabular}{|c|c|c|c|c|c|c|}
\hline Autor, año [referencia] & Tipo de estudio & $\begin{array}{l}\text { Cribado } \\
\text { de TBL }\end{array}$ & $\begin{array}{l}\text { Incluyó } \\
\text { TBL }\end{array}$ & $\%$ de TBL & $\begin{array}{l}\text { Inmunosupresores } \\
\text { concomitantes }\end{array}$ & Calidad de los estudios \\
\hline Andrade, $2014^{18}$ & Longitudinal & Sí & Sí & 26,7 & MTX, Cys & 5 de $6 *$ \\
\hline Cataño, $2016^{19}$ & Longitudinal & Sí & Sí & 100 & NR & 5 de $6 *$ \\
\hline Duarte, $2011^{20}$ & Longitudinal & Sí & No & 0 & NR & 5 de $6^{*}$ \\
\hline Gomes, $2015^{21}$ & Cohortes & Sí & Sí & 18,8 & CS, MTX, Lef & 7 de $8^{*}$ \\
\hline Labarca, $2003^{22}$ & Longitudinal & Sí & NR & NR & DMARD & 4 de $6 *$ \\
\hline Machado, $2013^{23}$ & Longitudinal & NR & NR & NR & DMARD, GC & 4 de $6^{*}$ \\
\hline Scali, $2005^{24}$ & Longitudinal & Sí & No & 0 & MTX & 5 de $6 *$ \\
\hline Valdés, $2006^{25}$ & Longitudinal & Sí & NR & NR & No & 5 de $6 *$ \\
\hline Valenzuela, $2017^{26}$ & Ensayo clínico & NR & NR & NR & No & $\begin{array}{c}\text { Bajo riesgo: } \mathrm{SA}, \mathrm{OA}, \mathrm{OPP}, \mathrm{RI} \text {; Riesgo no } \\
\text { claro: } \mathrm{ECR}, \mathrm{RS}^{* *}\end{array}$ \\
\hline
\end{tabular}

\% porcentaje, * escala de Newcastle-Otawa para estudios observacionales adaptada, ** herramienta de la colaboración Cochrane para evaluar riesgo de sesgos en ensayos clínicos. TBL: tuberculosis latente; MTX: metotrexato; PDN: prednisona; Cysp: ciclosporina; NR: no hay datos suficientes reportados; CS: corticoides sistémicos; Lef: leflunomida, DMARD: drogas antirreumáticas modificadoras de enfermedad; GC: glucocorticoides sitémicos; SA: secuencia aleatoria; OA: ocultamiento de asignación; OPP: ocultamiento a los participantes y personal; RI: resultados incompletos; ECR: evaluación del ciego de resultados; RS: reporte selectivo. 
las recomendaciones sobre el cribado y tratamiento de la tuberculosis latente en pacientes que van a recibir anti-TNF- $\alpha^{8}$.

Es preciso mencionar un posible subregistro de los casos en Latinoamérica de pacientes recibiendo terapia anti-TNF- $\alpha$ por psoriasis, dada la poca cantidad de estudios encontrados ${ }^{19}$ y que muchas veces no se reportan estos efectos adversos, aunque sí existen estudios heterogéneos sobre tuberculosis activa en pacientes que reciben terapias anti-TNF- $\alpha$, y otros biológicos, principalmente para artritis reumatoide, algunos reportados de modo global, sin poder hacer la diferenciación entre patologías $^{31,32}$. Además, es posible un subdiagnóstico de tuberculosis en los estudios seleccionados, dado que sólo uno de los 9 estudios reportó pacientes con tuberculosis, además de que algunos estudios tuvieron periodos de seguimiento cortos, incluso cercanos a las 12 semanas $^{22,26}, y$ en muchos casos los estudios fueron sobre seguridad, no especificando cual fue la estrategia activa para la detección de los casos de tuberculosis. En el caso del Perú, no se han encontrado a la fecha reportes de caso de tuberculosis en pacientes recibiendo anti-TNF- $\alpha$ por psoriasis $u$ otras indicaciones, pese al uso cada vez mayor de dicha terapia en nuestro medio $^{6}$, por lo que también es de esperar un subregistro.

\section{CONCLUSIONES}

La principal conclusión de la presente revisión es una incidencia combinada de tuberculosis activa en pacientes con psoriasis recibiendo anti-TNF- $\alpha$ de 636 por 100 mil pacientes/año en Latinoamérica (IC95\%: 145 a 1764 por 100 mil pacientes/año), la cual fue elevada si se le compara con población general de Latinoamérica, y que es similar a estudios de otras latitudes de población con psoriasis recibiendo dicha terapia.

Aún es preciso complementar dichos estudios con cohortes más grandes y de mayor tiempo de seguimiento, con mejor registro de los factores de riesgo y las estrategias para un cribado y manejo adecuado de la tuberculosis latente, así como para evaluar mejor el riesgo de tu- berculosis activa asociada a terapia antiTNF- $\alpha$, y también estudios que tengan un grupo de control o cohorte no expuesta en Latinoamérica, a fin de efectuar comparaciones y determinar riesgos.

\section{AGRADECIMIENTOS}

Al Dr. Juan Cataño y al Dr. Marcelo Pinheiro, por su colaboración en la elaboración de la presente revisión.

\section{REFERENCIAS BIBLIOGRÁFICAS}

1. World Health Organization. Global tuberculosis report 2018 [Internet]. Geneva: World Health Organization; 2018 [cited 2018 Oct 3]. Disponible en: http://www.who.int/tb/publications/global_report/en/

2. World Bank. Incidence of tuberculosis in Latin America and Caribbean [Internet]. Washington D. C.: World Bank; 2016 [cited 2018 Oct 3]. Disponible en: https://data.worldbank.org/indicator/SH.TBS INCD? locations $=Z \mathrm{~J}$

3. Deng Y, Chang C, Lu Q. The Inflammatory Response in Psoriasis: a Comprehensive Review. Clin Rev Allergy Immunol. 2016;50(3):377-89. DOI: 10.1007/ s12016-016-8535-x

4. Gupta R, Debbaneh MG, Liao W. Genetic Epidemiology of Psoriasis. Curr Dermatol Rep. 2014;3(1):61-78. DOI:10.1007/s13671-013-0066-6

5. IPC. Revisión del IPC sobre psoriasis [Internet]. Missouri: Intenational Psoriasis Council; 2009 [cited 2018 Oct 3]. Disponible en: http://www.psoriasiscouncil.org/docs/ipcpsoriasisreview_dec_2009_ spanish.pdf?LanguageID=EN-US

6. Bravo E, Bar N, Paucar S, Mendoza R. PASI reduction in patients with moderate-severe psoriasis treated with biological therapy. Experience in a Peruvian Tertiary Hospital. Med Cutan Iber Lat Am. 2016;44(1):41-4.

7. Lorenzetti R, Zullo A, Ridola L, Diamanti AP, Laganà $B$, Gatta L, et al. Higher risk of tuberculosis reactivation when anti-TNF is combined with immunosuppressive agents: a systematic review of randomized controlled trials. Ann Med. 2014;46(7):547-54 DOI: 10.3109/07853890.2014.941919

8. Gómez-Reino JJ, Carmona L, Angel Descalzo M Biobadaser Group. Risk of tuberculosis in patients treated with tumor necrosis factor antagonists due to incomplete prevention of reactivation of latent infection. Arthritis Rheum. 2007;57(5):756-61. DOI: 10.1002/art.22768

9. Garcia-Doval I, Cohen AD, Cazzaniga S, Feldhamer I, Addis A, Carretero G, et al. Risk of serious infections, cutaneous bacterial infections, and granulomatous infections in patients with psoriasis treated with anti-tumor necrosis factor agents versus classic therapies: Prospective meta-analysis of Psonet registries. J Am Acad Dermatol. 2017;76(2):299-308.e16. DOI: 10.1016/j. jaad.2016.07.039

10. Ergun T, Seckin D, Baskan Bulbul E, Onsun N, Ozgen Z, Unalan $\mathrm{P}$, et al. The risk of tuberculosis in patients with psoriasis treated with anti-tumor necrosis factor agents. Int J Dermatol. 2015;54(5):594-9 DOI: $10.1111 /$ ijd.12628

11. Cantini F, Niccoli L, Goletti D. Adalimumab, etanercept, infliximab, and the risk of tuberculosis: data from clinical trials, national registries, and postmarketing surveillance. J Rheumatol Suppl. 2014;91:47-55. DOI: https://doi.org/10.3899/ jrheum.140102

12. Stroup DF, Berlin JA, Morton SC, Olkin I, Williamson GD, Rennie D, et al. Meta-analysis of observational studies in epidemiology: a proposal for reporting. Meta-analysis Of Observational Studies in Epidemiology (MOOSE) group. JAMA. 2000;283(15):2008-12. DOI: 10.1001/ jama.283.15.2008

13. Ottawa Hospital Research Institute. The NewcastleOttawa Scale (NOS) for assessing the quality of nonrandomised studies in meta-analyses [Internet]. Ottawa: Ottawa Hospital Research Institute; 2018 [cited 2018 Oct 8]. Disponible en: http://www.ohri. $\mathrm{ca} /$ programs/clinical_epidemiology/oxford.asp

14. Higgins JP, Altman DG, Gøtzsche PC, Jüni $P$, Moher D, Oxman AD, et al. The Cochrane Collaboration's tool for assesing risk of bias in randomised trials. BMJ. 2011;343:d5928. DOI: 10.1136/bmj.d5928

15. Miller JJ. The Inverse of the Freeman - Tukey Double Arcsine Transformation. Am Stat. 1978;32(4):138. DOI:10.1080/00031305.1978.10479283

16. Dersimonian R, Laird N. Meta-analysis in clinical trials. Control Clin Trials. 1986;7(3):177-88. DOI: 10.1016/0197-2456(86)90046-2

17. Moher D, Liberati A, Tetzlaff J, Altman DG, PRISMA Group. Preferred reporting items for systematic reviews and meta-analyses: the PRISMA statement. PLoS Med. 2009;6(7):e1000097. DOI:10.1371/ journal.pmed1000097

18. Andrade LM, De Oliveira B, Pinto AC, Pedreira VR, Follador I, SantosMF. Anti- TNF- $\alpha$ therapy in the management of psoriasis: experience of a state referral center. An Bras Dermatol. 2014;89(3):43640. DOI: $10.1590 / a b d 1806-4841.20142613$

19. Cataño J, Morales M. Isoniazid toxicity and TB development during biological therapy of patients with psoriasis in Colombia. J Dermatolog Treat. 2016;27(5):414-7. DOI: 10.3109/09546634.2016.1151857

20. Duarte AA, Chehin FB. Moderate to severe psoriasis treated with infliximab - 53 patients: patients profile, efficacy and adverse effects. An. bras. dermatol. 2011;86(2):257-63. DOI: 10.1590/S036505962011000200008

21. Gomes CM, Terreri MT, Moraes-Pinto MI, Barbosa C, Machado NP, Melo MR, et al. Incidence of active mycobacterial infections in Brazilian patients with chronic inflammatory arthritis and negative evaluation for latent tuberculosis infection at baseline - A longitudinal analysis after using TNFa blockers. Mem Inst Oswaldo Cruz. 2015;110(7):921-8. DOI: 10.1590/0074-02760150235

22. Labarca C, Massardo L, García PI, Jacobelli S. Evaluación del tratamiento con infliximab en enfermos con artritis inflamatoria refractaria a drogas habituales. Rev Med Chil. 2003;131(10):1157-64. DOI: 10.4067/S0034-98872003001000009

23. Machado NP, Reis Neto ET dos, Soares MRMP, Freitas DS, Porro A, Ciconelli RM, et al. The skin tissue is adversely affected by TNF-alpha blockers in patients with chronic inflammatory arthritis: a 5-year prospective analysis. Clinics. 2013;68(9):1189-96. DOI: 10.6061/clinics/2013(09)03 
24. Scali JJ, Kaminsky A, Salomón J, Visentini S, Dancziger E. Open-label 24-month study evaluating infliximab therapy in patients with psoriatic spondyloarthropathy. Ann N Y Acad Sci. 2005;1051(1):543-50. DOI: https://doi.org/10.1196/ annals.1361.098

25. Valdés A. MDP, Schroeder H. F, Roizen G. V, Honeyman M. J, Sánchez M. L. Eficacia y seguimiento en el largo plazo de pacientes con psoriasis vulgar moderada a severa en tratamiento con infliximab (Remicade®). Rev Med Chil. 2006;134(3):326-31. DOI: 10.4067/S0034-98872006000300009

26. Valenzuela F, de la Cruz Fernandez C, Galimberti RL, Gürbüz S, McKean-Matthews M, Goncalves L, et al. Comparison of ixekizumab with etanercept or placebo in moderate-to-severe psoriasis: Subgroup analysis of Latin American patients in the phase 3 randomized UNCOVER-3 study. Actas
Dermosifiliogr. 2017;108(6):550-63. DOI: 10.1016/j. ad.2017.02.005

27. World Health Organization. Tuberculosis country profiles. [Internet]. Geneva: World Health Organization; 2015 [cited 2018 Nov 17]. Available from: http://www.who.int/tb/country/data/profiles/en/

28. Gisondi P, Cazzaniga S, Chimenti S, Maccarone M, Picardo M, Girolomoni G, et al. Latent tuberculosis infection in patients with chronic plaque psoriasis: evidence from the Italian Psocare Registry. $\mathrm{Br} \mathrm{J}$ Dermatol. 2015;172(6):1613-20. DOI: 10.1111/ bjd. 13130

29. Sánchez-Moya Al, García-Doval I, Carretero G, Sánchez-Carazo J, Ferrandiz C, Herrera Ceballos $\mathrm{E}$, et al. Latent tuberculosis infection and active tuberculosis in patients with psoriasis: a study on the incidence of tuberculosis and the prevalence of latent tuberculosis disease in patients with moderate-severe psoriasis in Spain. BIOBADADERM registry. J Eur Acad Dermatol Venereol. 2013;27(11):1366-74. DOI: 10.1111/jdv.12011

30. Centers for Disease Control and Prevention. Core Curriculum on Tuberculosis: What the Clinician Should Know. [Internet]. Atlanta: Centers for Disease Control and Prevention; 2013 [cited 2018 Nov 17]. Available from: https://www.cdc.gov/tb/ education/corecurr/pdf/corecurr_all.pdf

31. Vega MD la, Gómez G, Casado G, Battagliotti C, Exeni I, Gobbi C, et al. Tercer reporte de eventos adversos con tratamientos biológicos en Argentina. Informe de registro BIOBADASAR. Rev. argent. reumatol. 2013;24(4):9-14

32. Galvis L, Sánchez ÁY, Jurado LF, Murcia MI. Tuberculosis associated with tumor necrosis factor- $\alpha$ antagonists, case description and analysis of reported cases in Colombia. Biomédica. 2018;38(1):7-16. 